\title{
Ueber die Funktion der vertikalen Bogengänge.
}

\section{Von Dr. R. Schilling.} Die Erkenntnis, daß der Bogengangsapparat ein Sinnesorgan für
die Orientierung im Raume und die Regulierung des Gleichgewichts ist, veroanken wir Goltz (1870), welcher sich vorstellte, daB die in drei Dimensionen des Raumes angeordneten Bogengänge die Schwerkraft in drei Komponenten zerlegen und durch den Druck auf die Sinnesendstellen uns das Gefülll der Lage des Kopfes im Raume zum Bewußtsein bringen. Dieser statischen Auffassung der Bogengangsfunktion stellten $\mathrm{Mach}$ und $\mathrm{Breuer}$ später eine statischkinetische Theorie entgegen, nach welcher im Vestibularapparat zwei Sinnesorgane vereinigt sind, von welchen das eine, der Statolithen. apparat (Sacculus und Utriculus), uns die Lageempfindung im Raum und außerdem die Progressivbeschleunigung vermittelt, das andere aber, die Bogengänge, auf die Winkelbeschleunigung bei der Drehung reagiert und uns diese als das Gefühl der erlangten Winkelgeschwindig. keit zum Bewußtsein bringt.

Auch heute noch stehen wir auf dem Boden der Mach, Bréuer. schen Theorie, wenn auch eine Reihe von Fragen aufgetaucht ist, welche durch sie noch nicht oder nicht vollständig beantwortet werden.

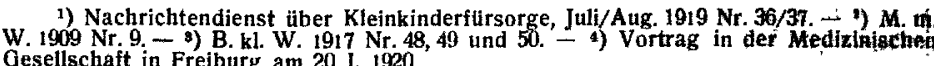
Gesellschaft in Freibury am 20. I. 1920 . 
Mit einigen dicser Fragen, so der physikalischen Kräfteıcrteilung in beiden Labyrinthen, der Funktion der vertikalen Bogengange und des Zusammenwirkens der beiderseitigen Bogengänge, habe iclı mich im Laufe der letzten Jahre beschäftigt und móchte lieute insbesondere über das Ergebnis meiner Untersuchungen über die vertikalen Bogengängc berichten.

s sind hauptsächlich zwei Erscheinungen, wclche durch Reizung der Bogengänge ins Leben gerufen werdett, erstens die subjektiven Empfindungen der Drehung und Scheindrchung und zweitens die objektiv wahrnehmbaren Reaktionsbewegungeıl der Augen, des Kopfes, des Rumpfes und der Extremitäten. Die erstıren sind wcgen der den subjektiveı Erscheinungen überhaupt anhaftenden Schattcnseiten und ihrer tatsächlichen Inkonstanz weniger geeignet für exakte Unter-suchungen, während hierfúr die oszillatorısclıen Bewegungen der Bulbi als das genaueste, konstanteste und mcßbare Reagcns auf die Bagengangs reizung 'stets bevorzugt worden sind.

Die ältestbekannte Bezeichnung zwisclien Nystagmus und Bogengangsreiz betrifft eine Bcobachtung, welche sclion Flourens in tung eines allgemein anerkannten Gesetzes verdichtet hat. Sie besagt, dali der Nystagimus in der Ebene des gereizten Bogengangs schlägt. Die Anwendung dieses Gicsetzes stöbt auf keitte Schwierigkeiten, wenn wir die horizontalen Bogengángc ins Auge fassen. Denn der horizontale Nystagmus sclulagt in der beiden Augenachsen gemeinsanen und nit der Horizontalebene des Schädels übereinstimmenden Ebene, in welcher annahiernd aucl dic horizontalen Bogengänge liegen. Allerdings bedarf das Flourensschc Gesetz auch hier sclıon einer Einschränkung, indem in Wirkliclıkeit die horizontalen Bogengänge sich nach vorn und seitlich über die Horızontalebene des Schädels erheben, bei der Taube nach Ewald um $20^{\circ}$, beim Menschen um $15-30^{\circ}$.

Sehr viel komplizierter gestalten sich diesc Verhaltnisse jedoch für die vertikalen Bogengänge. Denn die für die vertikalen Bewegungen der Bulbi in Betracht kommenden Ebenen gehören nicht einer gemeinsamen Ebene an, wie das bei den horizontalen Bewegungen der Bulbi der Fall ist, sondern bilden unter sich und mit der Medianebene Winkel, welche in weiten Grenzen schwanken ${ }^{1}$ ). Der Winkel, welchen die Augenachsen und danit auch die Schlagebenen des vertikalen Nystagmus mit der Medianebene bilden, kann in der Säugetierreihe alle Werte haben ron $5^{\circ}$ bis $85^{\circ}$. Beim Menscheri betragt $z$. B. dieser Winkel, cigentlich der latibe Hornhautwinkel, nach Pütter $5^{\circ}$, beim Löwen zirka $15^{\circ}$, beim Pferd $55^{\circ}$, beim Ameisenfresser $60^{\circ}$, beim Wasserschwein $75^{\circ}$. Bei den meisten Fischen und Vögeln beträgt er $80-85^{\circ}$.

Atuch der Wiukel, welchen die vertikalcn Bogengänge mit der Medianebenc bilden, schwankt bei verschicdctien Tieren acmlich stark. Er beträgt bei der Taube nach Ewald zıemliclı genau $45^{\circ}$ nach $\mathrm{H}$ as s e jedoch ist der obere Bogengang mehr der Sagittalebcne, der lintere mehr der Frontalebcue parallel. Nach einer Zeichnung Breuers beträgt bei der Meise der erstgenannte Winkel $50^{\circ}$, dcr letztgenannte $30^{\circ}$. Nach Denker fallen beim Wasserschwcin und Ameisenfresser die oberen Bogengänge nahez:11 mit der Medianebene, die hinteren mit der Frontalebcne zusanınen, während bcimı Lcoparden die oberen Bogengänge der Frontalebene, die hinteren Bogengänge der Medianebene stark genähert sind.

Wollten wir das Flourenssche Gesctz auf diese mannigfachen, in der Natur vertretenen Möglichkeiten genau anwenden, so würde dies zu durchaus verschiedenen Ergebnissen führen. In ciftem Falle, wo die Augenachsen sowohl als auch die Bogengange cincn Winkel von $45^{\circ}$ mit der Medianebene bilden, müßßte dic Reizung eines vertikaleı Bogenganges in dem eineı Auge vertıkalcn, im andern rotatorischen Nystagmus erzeugen. Da, wo die Augenachsen und die Bogengange mit der Median- bzw. Horizontalebene nahezu zusamnenfallen, müßte die Reizung cines vertikalen Bogenganges in beiden Augen gleichartigen Nystagmus (beim Wasserschwein z. B. die Rcizung eines hinteren Bogengangs in beiden Bulbi vertikalcn Nystagmus) hervorrufen.

Diese, auf anatomischer Grundlage gewonnenen Erwägungen zeigen, daß eine konstantc Beziehung zwischon Bogengangsstellung und Augenachsenwinkel nicht besteht, und lassen die volle Gültigkeit des F lo urens schen Gesetzes für die vertikalcn Bogengänge als zweifelhaft ersclicinen.

Welche Antwort geben uns nun dic tierexperimentellen Untersuchungen? Kubo hat im Jahre 1905 und 1906 am Kreidlschen Physiologischen Institut in Wien an Kaninchen und Tauben und an der Zoologischen Station in Triest an Fischen (Haffischen, Rochen, Torpedos u. a.) ausgedchnte Bogengangsexperimentc vorgenommen indem er die freigelegten Bogengänge teils thermisch mit kalten und heiBen Stäbchen, teils mechanisch mit enner Pinzettc oder Borste reizte. Während die Reizung der horizontalen Bogengänge bei allent Versuchstieren horizontalen Nystagmus erzeugte, war das Ergebnis der Reizung der vertikalen Bogengange sehr verschieden. Auf thermische Reize reagierten die Bogengängc dcr Fische fast gar nicht. Auf mechanische Reizung erfolgte bei sämtlichen Fischen bei Rcizung des vorderen Bogengangs eine Ruckbewegung des nasalen Bulbuspoles nach oben hinten, bei Reizung des hintercn Bogengangs derselben Seite eine Ruckbewegung desselben Poles nach unten rorne,

1) Angaben uber digse Winkel sind in der Literatur nur sehr sparlich vorhanden Bei Capybara und Myrmikophoga jubata konnte tch dank der Liebenswurdiykeit von Hern Prof. Ficcher diesen Winkel messen, soweit dies am makerierten Schadel uberhaupt moglkh ist. also beide Male in einer (diagonalen) Ebene, welche mit der Vertikalebene der Augenachsen einell gewissen Winkel bildet Manchmal waren diese Beilegungen auch rotatorisch, also senkrecht zur Bulbusachse.

Beim Kaninchen fielen de Reaktionen an den rertikalen Bogengangen sehr verschieden aus, bald tiaten horizontale, bald vertikale, ld Rollbewegungen, bald diagonale Bewegungen der Bulbi auf.

Die konstantesten Resultate ergaben die Versuche an Tauben. Bci ihnen erzeugte dic Rcizang des vorderen Bogenganges rotatorischen, dıe Reizung des hinteren Bogengangs rertikalen Nystagmus.

Die konstantestcr Resultatc zeigien demnach Tiere, welche auf ein feill arbeitendes Glcichgewichtsorgan angew'iesen sind, wie die Fische und besonders die Vogel; schwankende Resultate dagegen die auf ihr Glcichgewichtsorgan weniger angewiesenen Kaninchen. Keineswegs zeigen aber die Versuche eine durchgelıende Uebereinstimmung voll Bogengangsebene utid Schlagebene des Nystagnuts.

Beı Tiere"l mit parallelstehende" Augen sind isolierte Bogengangsrcizungen noch miclit gemacht wordin. Auch beim Menschen ist es bis jetzt noch nicht gelumgen, de vcrtikalen Bogengänge isoliert zu reizen. Vom horizontalen Bogengang wissen wir durch die Drehversuche, daß er horizontalen Nystagnius erzeugt. Von den vertikalen Bogengangen ist uns zwar aucli bekannt, da $B$ der durch sie bedingte Nystagmus in eincr vertikalen Ebcne schlagt. Wir wissen aber nicht, auf welchell vertikalen Bogengang die bekannten Hauptformen des vertikilen Nystagmus jewells zu beziehen sind, namlich der in der Lätıgsaclıse der Bulbi sclılagende, gemeinhin als vertikaler (besser sagittaler) bezeichncte Nystagmus und der in der Frontalebcne des Schadels schlagende, uin dıc Lälngachse des Bulbus rotierende rotatorisclie Nystagmus. Auch die Entdeckung des kalorischen Nystagmus durch Bárány, dic uns cine isolierte Labyrinthreizung crmoglichte, hat die Hoffnung, durch sie viclleicht noch cine weitere Differenziertung und isolierte Bogengangsreizung fu crzielen, nicht erfüllen können, wohl aber gibt sie unı einell Hinweis auf die Lösung dieser Frage. Da wir mit dem Temperaturgefälle nur den horizontalen und gleichzcitig meist noch die Ampulle des oberen Bogengangs erreichen, kaum aber den hinteren Bogengang, der durch cine dicke Gewcbsschiclit von der Abkühlungsfläche getrennt ist, und da wir bei der kalorischen Prüfung stcts horizontalen oder rotatorischen oder gemisclit rotatorisclihorizontalen, niemals aber vertikalen Nystagmus erhaiten, so liegt es nahe, den vertikalen Nysiagmus per cxclusionem dem hinteren Bogengang und, bei der feststehenden Funktion des horizontalen Bogengangs, den rotatorischen Nystagmus dem oberen Bogengang $7 \mathrm{u}$ uschreiben.

In einigen Fällen gelang es mir, diese beiden Funktionen zu trennen, indem der in Optimunistellung des horizontalen Bogengangs erzeugte, gemischt liorizontal-rotatorisclic Ny'stagmus beim Uebergang in die Pessimumstellung einem reinen rotatorischen Nystagmus l'latz. machtc, was wahrend derselben Prufung mehrmals wiederholt werden konnte.

Ich habe nun versucht, dieser Frage noch auf einem anderen Wege, nämlıch durch Drehversuche an einseitig Labyrinthlosen, nalıer zu konımen. Bringt man den Kopf in eine Stellung, iu welcher zwci gekreuzt korrcspondierende Bogengange in die Drehebene zu liegen kommen, eitre Stellung, die ich als schräge Kopfseitenlage ${ }^{1}$ ) bzw. schräge Kopfbeugclage ${ }^{2}$ ) (im Arch. f. Ohrhlk. 104 S. 120) nähcr beschrieben habe, so wird. da eine Seite unerregbar ist, nur c 1 n Bogen. gang der gestundell Seite bet der Drehung gcreizt, z. B bei linkseitiger Labyrınthlosigkeit in recliter scliriger Kopfseitenlage der rechte obcre Bogengang, in linker schräger Kopfseitenlage der rechtc hintere Bogengang. So fand ich in den untersuchten 8 Fällen, daß durch Reizung des obcren Bogengangs rotatorischer, durcli Reizung des hinteren Bogengangs vertikaler Nystagmus erzeugt

Nur in einem Fall trat in Kopfseitenlage, auch bei Einstellung des oberen Bogengangs in dic Drehebene, vertikaler Nystagmus auf, cin Verlalten, für das ich keine Erklarung weiß. - Die Richtung der langsamen Komponente entspricht auch hier der Endolymphstromungsriclıtıng. Die Stärke und Dauer dieses Nystagmus ist meist sehr gering, er zeigt manchmal nur wenige Schlage.

Wenden wir tuns nun al der Fragc des Zusammenwirkens der Bogengange beider Seıtell, so ist die allgemein herrschende Auffassung die, da $\beta$ bei der genıcinsamen Drehreizung beider Labyrinthe zwei gekreuzt korrespondierende Bogengänge weil in einer Ebene liegend zusammenwirken.

Auf Grund meiner Nystagnusbeobaclitungen komme ich zu eiment anderen Ergcbnis. Ich schließe, daß bei der Drelumg awci glcichnamig korrcspondierende Bogengätnge zusanımenwirken, und Zwar unter Ausschaltung der Wirkung der übrigen Bogengainge. Diese Auffassulng stitzt sich auf folgende Punkte:

1. Auf die weitcre Verfolgung der für die cinzelıen vcrtikalen Bogengänge gefundenen Funktionen. Drehen wir die Versuchsperson iII einer der Hatiptebenen des Kopfes, der inedianen oder der frontalcn Ehene, so werden alle vier vertikalen Bogengange, die ja nit jeder dieser Ebenen einen Wink:l ron $45^{\circ}$ bilden, physikal.sch gleich stark gereizt. Da mach den obigen Auseinandersctzungen das obere Bogcugangspaar rotatoriscleet, d"'s hintere rcrikalcn Nystagmus erzcugt, so mübtc man, wenı die Wirkuıng aller vier Bogengänge zur

R. R. K. S $=$ Kopf um $45^{\circ}$ nach links und $900^{\circ}$ nach der rechten Selte veneigt. - 
Geltung käme, ein Gemisch von rolatorischem und vertikalenı $\mathrm{Ny}$ stagnius als Ergebnis erwartell. Die Erfalnung zeigt aber, daß wir bei Drehung in der Mediancbene stets reinen verikalen, bei Drehung in der Frontalebene stets reine!n rotatorischen Nystagnuus erlialten Wie erklart sich dieser W/iderspruclı? W/ir müssen hier eilı zweites experimentell erwesenes Gesetz zu Hilfe nehmen, welches die Schlagrichtung des Nystagintus betiffit und besagt, daf die langsame Komponente des Nystagnius gleicligerichtet ist der Richtung der Endoympistrómtung:

Drehen wil den Kopf in der Frontalebene, (un seine sagittale Achse) nach redits - z z. B. in gerider Kopfbetgelage -, so bewegt sich bein Beginn der Drchung die Endolytuplie in dell beiden hiulteren Bogengä̈ıgen nach links, und zwar im rechıten hinteren Bogengang vou rechts hinteu uber oben nach lukk vorne (ampullofugal). Diese Endolyniplibewegung eizengt vertikalen Nystagnus nacli aufwarts (die langsane Komponente entsprechend der Nystagmusriclıtung nach abwarts, die rasche naclı autwärts). Im linken hinteren Bogengang sirömt die Endolymphe von reclits vorn tiber oben nach links hinten (ampullopetal) und erzcugt vertikalen Nystagmus nach abwärts. Beide Nystagmusbewegungen mussen sich aber aufheben, da sie gleich stark und entgegcngesetzt gerichtet sind. Es bleibt also nitr noch die Wirkung der Endolymphstromung in den oberen Bogengangen übrig. Diese bewegt sich inn rechten oberen Bogengang von reclits vorne über oben nach links hinten (anıpullofugal) und erzeugt rotatorischen Nystagulus nach rechts, inı linkclı oberen Bogengang von rechts linten uber oben nach links vorne (anpullopetal) und erzeugt ebenfalls rotatonischen Nystagmus nacli rechits. Beide Wirkungen addieren sich also und ergeben als Endeffekt, wie die Eriahrung bestätigt, rotatorischen Nystagnilus nach rechits.

Drehlen wir den Kopf in der Medianebene (um seine transversale Aclise) nach vorne oder, was das Gileiclie ist, in linker gerader Kopfseitenlage nach rechits, so bewcogt sich zu Beginn der Drehung die Endolynplıe in beiden oberen Bogengängen anipullofugal nach innen und hinten gegen die Medianebene des Kopfes zu. Sie stromt also it beiden Bogengängen in entgegengesetzter Richtung, im rechten nach links linten, im linken nach rechis hinten. Die erstere erzeugt rotatorischen Nystagmus nach rechis, die letztere erzeugt rotatorisclicn Nystagmus nach links. Beide Nystagmusbewegungen müssen sich, da sie entgegengesetzt gerichtet sind, gegenseitig auflıeben. Es bleibt dennaclı nur noclı der Effekt der Elıdolymphströnung jul den linteren Bogengängen übrig. In diesen beiden Bogengängen stromt sie ampullopetal, von der Medianebene weg nach hinten und außen. Die Endolyniplibewegung inl recliten liinteren Bogengang erzeugt vertikalen Nystagnıus nacl abwärts, diejenige in linken linteren Bogengang ebenfalls vertikalen Nystagmus nach abwärts. Beide Nystagm»sbewegungen addieren sich $\mathrm{zu}$ dem Endcffekt: vertikaler Nystagnius naclı abwärts, wie das Experinlent bestätigt.

2. Gegen dic Annalıme des Zusammenwirkens zureier gekreuzt korrespondierender Bogengänge sprechen auchı die Reaktioneı, die nlan bei gleichzeitiger Reizung dieser Bogengänge erlıält. Wäre ein gekreuztes Bogengangspaar dazu bestimnit, zusammen zu wirkell, so sollte man erwarten, daß man bei ihrer gleiclizeitigen Reizung in physikalisclı günstigster Stellung deutliche, gleiclısinnige Nystagmusrealitionen erlielte. Das ist aber in der Tat nicht der $F$ all. Icli habe bei einundzwanzig beiderseits labylnthlgesunden hndwiduen versucht, die gekreuzt korrespondierenden Bogengänge durch ilıre Einstellung in die Drelıebene in den sclırägen Kopfstellungslagen, die ichı auch ats plyysikalische Optimunstëllung bezeichnet habe, zu reizen.

Die dabei bcobachteten Nystagmusrealitionen standen sowolil flinsiclıtlichı ihırer Intensität und GleichnäBigkeif als auch ilırer physiologisclıen Zwecknı̈ß̈ßigkcit weit linter den Reaktionen zurück, welchic wir bei Drehung in den Hauptebenen des Kopfes, die ich als physiologische Optimumstellung bezeiclnet liabe, beobacliten. Das Ergennis nieiner schon vor 10 Jaliren begonnenen Drehversuche wat sogar so mannigfaltig und vielgestaltig, daß jch sie damals als unentwirtbar wieder aufgegcben liabe. SchlieBliclı ist es mir aber doch gelungen, in ilmen eine gewisse Gesetzmäßigkeit zu erkeınen und sic in mehrerc, unter sıcli gleicliartige Gruppen eimzuteilen. Ich will auf die genauere Darstellung dieser Gruppen (1. c.) hier nicht nälıer cingehen, sondern nur hervorlıeben, daß sie sich in der Hauptsache durclı das Ewaldsche Gesetz der Wcrtigkeit der Endolymphstronıungsiclitung und durch die anatomische Varietät der Bogengangsstellungen erklären lassen. Nach den Ewald schen Versuchen mit den pnemunatisclien Hammer sind die beiden Endolymph. stromungsrichtungen nicht gleichwertig, sondern ist dic anlpullopetale Strömungsrichtung die wirksamerc. Andere Autoren kamen zu anderen Ergebuissen, die meisten stimmen aber darin überein, daß eine Richtung wirksamer ist als die andere. Stehen die beiden in die Drehebente eingestellten, gekreuzt korrespondiercnden Bogengänge unter gleiclien pliysikalischen Bedingungen - was dann der Fall ist, wenn sie nit der Mednanebene wirklich einen Winkel von $45^{\circ}$ bilden -., dann liegt die Entschieidung, welche der beiden Reaktionen, ob vertikaler oder rotatorischer Nystagnius zum Vorschein konmt, nur noch an der Wertigkeit der Endolymphströmungsrichtung, indem die wirksamere Richtung - in meinen Fällen meist die ampullopetale Strönungsincitumg - überwiegt. Dadurclı würden sich restlos diejenigen Fälle - es waren deren vicr - erklären, bei welchen ich in allen sclurägen Kopfstellungslagen bei der Drclung in der einen Richtung rotatorisclien, bei der Drehing in der anderen Richtung vertikalen Nystaguntus erhalten liahe. Die ge inge Anzalıl dieser - den theoretisclien Voraussetzungen vollauf cutsprechenden - Re- sultati erklärt sich aus der Tatsache, daß nur sehr wenige' Schläfenbeine, wic aus dell Scliöllenla anll schien Messungen zuberechmen ist, dieses regelrechte Verhalten zeigen. Die meisten Schläfenbeine zeigen ein mohr oder weniger starkes Abweichen von der Winkelstellung von $45^{\circ}$ ilırer vertikalen Bogengäıge. Betraclıten wir, unı nur cin Bcıspiel anzufulıreı, den Schadel 36 der Sch ö 11 e Tabelle, ber welchenl dic oberen Bogengänge nit der Mcdianebene cinen Winkel von $45^{\circ}$, die hintcren einen solchen von $20^{\circ}$ mit der Medianebenc bilden, so wird, wenl wir ein solches Bogengangspda1 in die versuchte Optinunistellung bringen, immer nur e in Bogengang, in diesem Falle ein obercr, wirklich in Optinumstellung gelangen, der anderc jedoch, durch seinc Neignng von $25^{\circ}$ gegen die Dielıebene stets hinsichtlich dcr Strömungsenergie sciner Endo-
lymplie in Nachteıl sein. Es wird also bei allen Drelumigen überwiegcnd nur die Reaktion des in Optinuminsteliung befindlichen Bogengangs, in diesen Falle also rotatorischer Nystagmus zu erwarten sein. En11 solches Verlalten zeigt aber eine Gruppe von Fällen, bei welchen in allen sclırigen Kopfstellungslagen und in jeder Dreltrichtung nur otatorisclier Nystagnitis elitsland. Achnlich lassen sich auch die meisten anderen Gruppen durch Konkurrenz der Endolymphstıönungswertigkeit und der anatonischen Stellung der Bogengänge erklaren. Auch die asy'munetrischen Schläfenbeine finden thre Parallele in den resultierenden Nyslagmusforlnen ciner Gruppe.

3. Das physiologische Eingestelliscin der gleichnamig korrespondierenden vertikaleı Bogengünge findet seinen anatonischen Ausdruck in slurer Winkelbezichung zur Medianebene. Nach der Schön ein a n 11 schen Tabelle zeigen die gleichnanigen vcrtikalen Bogengange in mehr als der Halfte der Fälle eill streng symmetrisclies Verhalten zur Medıanebene, wäırend d:e gekreuzt korrespondierenden Bogengänge nur in einem einzigen Falle wirklich in der gleichen Ebene liegen. Die symunctrische Lage der vertikalen Bogengangspaare würde aber Inur dann eine gleiclıe pliysikalısclie Kräfteverteilung in den Bogengäıgen beider Labyrintlıe - wic sie die oben aufgestellte Theoric annimmt - gewährleisten, wenn tatsächlich in beiden Laby-
rintlien bci der Drelıung die gleiche Kraft wirksanl ist. Es wird vou andcrer Seite die Auffassung vertretell, dab im exzentrisch gelagerten Labyrinth die größere Kraft wirke. Iclı bin auf Gruind von mathenuatischen Ueberlegungen zu der Auffassung gelangt, daß in beiden Labyrinthen, unabliängig von ilırer Lage zun Drehpunkt, die gleichen Krifte wirksam sind (l. c.).

Zusammenfassend ließe sich demuach sagen: $D$ as Flourenssclie Gesctz läßt"sicl aul dievertikalcn Bogengänge niclit strikte anwenden, sondern, unabhängig vonder individuell schwankenden Bogengangsstellung, is der rotatorisclic Nystagmus beinl Meuschen eine Funk tion der oberen, der vertikale Nystagmus eine Funk. tion der li isteren Bogengänge.

Bei Drehungen ill den Hauplebcncudes Kopfes. (ge raden Kopfstellangslagenl, welche gleichzeitig die plysiologisclie Optimumstcllung sind, wirken jezwei gleichnanige vertikale Bogengängc unter Ausschluß der andercnzusanmen. Bei Drehungen in den schrägen Ebenen des Kopfes (schrägen Kopfstellungslagen), wclche gleichzeitig dic plysikalische optimumstel. lung silld, komnit von jc zwei gekreuzt korrespondie. readen Bogengängen meist nurdıe Wirkung des einen 7nr Geltullg, ulld zwar in Ablü̈lgigkeit vonder Wirk samkeit der Endolymphströmungsriclitung und der anatomisclie $\operatorname{Varietät}$ der Bogengangsstellung.

Wenn wir in dem Nystagmus eine Einrichtung sehen, welche die optische Verschiebung der Gegenstände bei der Drehung gleichsam wie ein Stroboskop verlangsanit und der Entstehung von Scliwindel dadurch enttgegerıwirkt, so ist die Vollkomminenlieit dieser Einrichtung beschränkt auf die Hauptebenen des Kopfes und auf ein individuell verschieden breites Ebenenbündel zu beiden Seiten dieser Hauptebeneı. Iı deı sclıägen Ebenen des Kopfes ist sie aber unvollkommen und läßt die physiologisclie Zweckmäßigkeit mehr oder weniger vermissell. - Die Diskrepanz zwischen der Nystagmusreaktion in physiologisclier und pliysikalischer Optinıunıstellung fordert eine Erklärung. Ist die Nystagmusreaktion lediglicl yon der Bogengangsreizung abhängig, dann ist die fast rudimentäre Reaktion in physikalischer Optimumstellung schwer verständlich. Es liegt desIalb der Gedanke nalıe, noclı einen anderen, hauptsächlich nur voll der Kopfstellung abhängigcı Reiz als wirksam anzunelınien, unı so mehr, als auch bei einseitig Labyrintllosen ein großer Unterschied in den Nystagmusreaktionen der schrägen und geraden Kopfstellungslagen zuguisten der letzteren sich ergeben liat. Ein solclier, in seiner Stärke von der Kopfstellung abhängiger Reiz ist aber der Makula reiz, der auch wälırend der Daucr der Dreliung durch die Wirkung offenbar einen Einflu $\beta$ auf die zentrale Verwertung des Ampullenreizes ausubt. Naclı den experimentellen Untersuchurgen von $K u b$ o bestelit eine enge und komplizierte Beziehung zwischen Makula und Ampulle. Anatonisch findet sie ilıren Ausdruck in den Verzweiguingen des Nervus vestibularis, durclı welchen die Macula utriculi und die Ampulle des horizontalen und oberen Bogengangs einerseits und die Macula sacculi und die Ampulle des hinteren Bogengangs anderscits in engerer Beziehung stehen. Es mußte danacl in Kopfbeugelage die Macula utriculi, in Kopfseitenlage die Macula sacculi bei der Drehung stärker gereizt werden. 
Die Annahme eines Einflusses des Makula reizes auf die zentrale Verwertung des Ampullarreizes würde auch die physiologische Summation des Drehreizes erklären. Wir müssen uns den der Ampullarreizung zugrundeliegenden physikalischen Vorgang als äußerst kurz dauernd vorstellen, da die Endolymphbewegung durch die Kapillarität des Bogengangslumens sofort aufgehoben wird. Würde die Stärke und Dauer des Nystagmus nur vom Ampullarreız abhängen, so wäre die bedeutend längere Dauer des Nystagmus nach mehreren gleichmäßigen Umdrehungen gegenüber der nach einer Umdrehung schwer zu verstehen; sie wird meines Erachtens bessser erklärt durch die Mitwirkung eines Reizes, der während der ganzen Dauer gleichmäBiger Umdrehungen weiter wirkt, wie dies beim Makulareiz der Fall ist. Sollte sich diese Auffassung bestätigen, so müßte die $\mathrm{Mach-Breuersche}$ Theorie, welche zwischen der Statholithenfunktion und der Ampullenfunktion eine scharfe Trennung vornimmt, dahin modifiziert werden, daB zwischen beiden Organen hinsichtlich der Nystagmusreaktion doch eine engere Beziehung besteht. 